\title{
Coulisses
}

Revue de théâtre

8 | Eté 1993

Varia

\section{Théâtre Universitaire de Vilnius}

\section{(2) OpenEdition}

1 Journals

Édition électronique

URL : http://journals.openedition.org/coulisses/2561

DOI : $10.4000 /$ coulisses. 2561

ISSN : 2546-9460

Éditeur

Presses universitaires de Franche-Comté

Édition imprimée

Date de publication : 1 juillet 1993

Pagination : 24-25

ISSN : 1150-594X

\section{Référence électronique}

"Théâtre Universitaire de Vilnius », Coulisses [En ligne], 8 | Eté 1993, mis en ligne le 15 mars 2019 consulté le 22 octobre 2019. URL : http://journals.openedition.org/coulisses/2561 ; DOI : 10.4000/ coulisses. 2561

Ce document a été généré automatiquement le 22 octobre 2019.

Coulisses 


\section{Théâtre Universitaire de Vilnius}

\section{Le metteur en scène}

\section{Alge Savickaite}

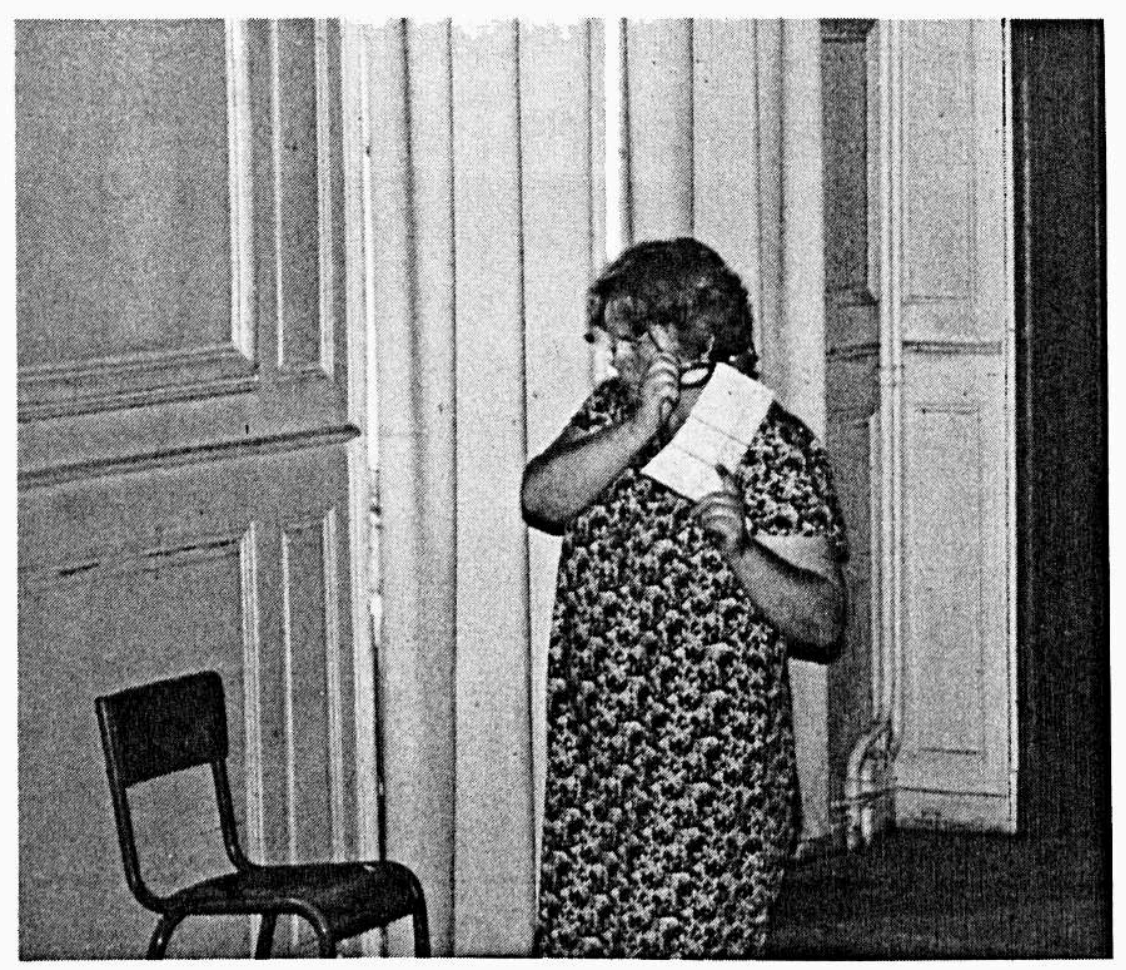

\section{Son point de vue}

1 Les thèmes essentiels dans la pièce de Shakespeare Cymbeline sont les thèmes du pardon et de la réconciliation. Les pays d'Europe occidentale qui, par le passé ont souvent guerroyé les uns contre les autres, entretiennent facilement des relations économiques. 
De leur côté, les pays d'Europe orientale, en devenant toujours plus libres, se souviennent aujourd'hui des anciennes offenses, politiques ou morales. Espérons que cela n'ira pas jusqu'à la confrontation.

2 L'idée de notre participation repose sur l'impossibilité de s'isoler dans ce monde-là. Tôt ou tard, il faut rencontrer ses voisins, accepter ou refuser les relations qu'ils proposent, s'adapter. C'est le destin de chaque personne dans une société et de chaque pays au sein de la communauté des Etats.

3 La tolérance et la compréhension sont nécessaires pour que la coopération soit possible. Il faut avoir du respect pour le caractère propre de chaque membre, pour l'histoire, la langue, la psychologie, les mœurs et la religion d'une nation. En résolvant les conflits, on doit se souvenir de la mort qui est la même pour tout le monde. Devant elle, on pardonne aux ennemis, on a de la pitié pour son prochain. Et on doit vivre selon ce principe.

4 En approchant d'une nouvelle époque de coexistence pacifique, on doit se « décrasser ». L'Europe, berceau de traditions culturelles anciennes pourrait devenir l'exemple moral de la paix permanente.

5 Restons donc fidèles à nous-mêmes et soyons unis !

\section{Les étudiants sur scène}

6 Arnoldas ALISAUSKAS

Saulius CIEPLA

Evaldas DIRGINCIUS

Jurga GAILIUTE

Giedre GANATAUSKAITE

Zilvinas JONUSAS

Rita KAZAKEVICIUTE

Jurgita ORLOVAITE

Darius RAKAUSKAS

Saule SARPYTE

Dainius VENGELIS 


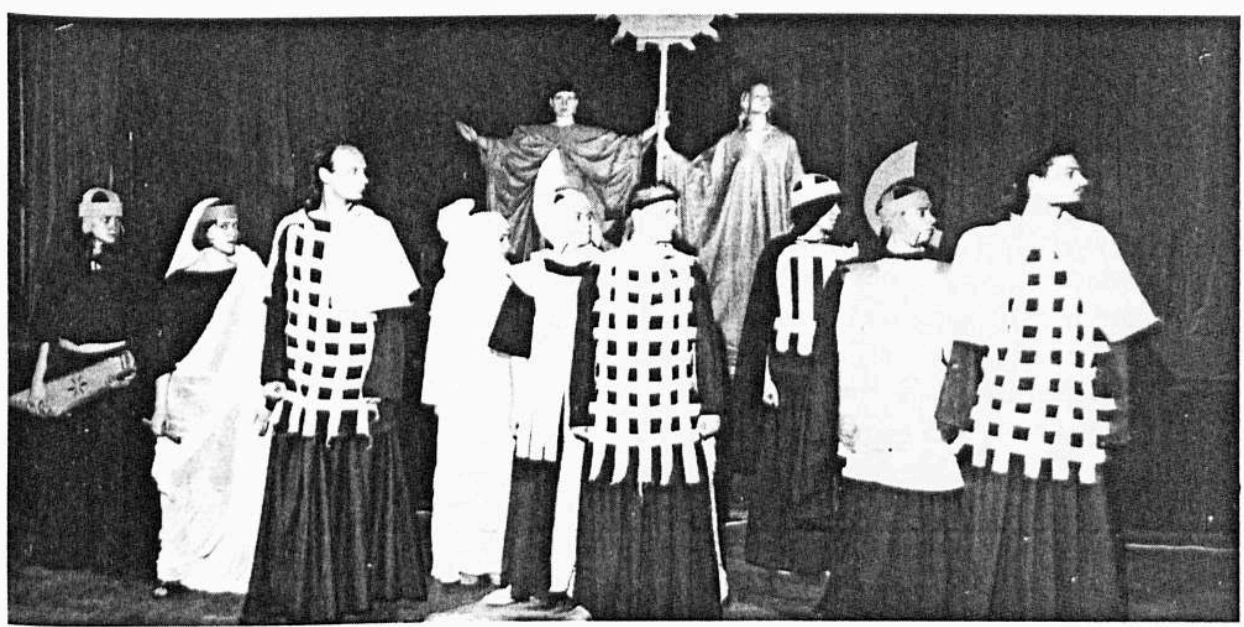

\section{Le T.U.}

7 En Lituanie, la tradition du théâtre universitaire est très ancienne et très vivante. Il y a plus de quatre siècles, le 18 octobre 1570 très exactement, que s'est joué en latin le premier spectacle produit par un groupe d'étudiants dans une des cours de l'Université de Vilnius pour célébrer la rentrée universitaire. Comme ce mode de représentation en plein air dans les différentes cours de l'Université se perpétua, c'est tout naturellement que le premier théâtre universitaire de Vilnius prendra le nom de Théâtre de la Cour de l'Université.

8 Depuis le début des années 90 , en même temps que la Lituanie retrouve sa liberté, on voit se multiplier dans tout le pays le nombre des théâtres universitaires dans le cadre de l'Association des Théâtres Libres.

9 Selon sa présidente Stase Luksiene, l'association a pour but principal de "stimuler l'initiative artistique des théâtres, d'organiser des festivals locaux et internationaux, de participer aux projets et festivals étrangers, de populariser les traditions du théatre et de la culture lituanienne à 1'étranger et de faire connaître au public lituanien les théâtres amateurs étrangers. »C'est grâce à son aide que l'on a pu voir des productions lituaniennes dans plusieurs festivals de théâtres universitaires: Innsbruck, Liège, Besançon, Paris, Londres, ...

10 Aujourd'hui, c'est une nouvelle troupe universitaire qui participe au projet Cymbeline. Elle réunit des étudiants en philologie de l'Université de Vilnius qui se destinent après leurs études à une carrière d'acteur professionnel au sein du Théâtre du Vieux Vilnius. 